\title{
Numerical investigation on the initiation of oblique detonation waves in stoichiometric acetylene-oxygen mixtures with high argon dilution
}

Yuhang Zhang ${ }^{\mathrm{a}}$, Yishen Fang ${ }^{\mathrm{b}}$, Hoi Dick $\mathrm{Ng}^{\mathrm{c}}$, Honghui Teng ${ }^{\mathrm{a}, *}$

a School of Aerospace Engineering, Beijing Institute of Technology, Beijing 100081, China

${ }^{\mathrm{b}}$ State Key Laboratory of High Temperature Gas Dynamics, Institute of Mechanic, Chinese Academy of Sciences, Beijing 100190, China

${ }^{c}$ Department of Mechanical, Industrial and Aerospace Engineering, Concordia University, Montreal, QC H3G 1M8, Canada

\section{A R T I C L E I N F O}

\section{Article history:}

Received 25 August 2018

Revised 30 January 2019

Accepted 22 March 2019

\section{Keywords:}

Oblique detonation

Acetylene-oxygen mixture

Argon dilution

Initiation structure

\begin{abstract}
A B S T R A C T
Oblique detonation waves (ODWs) in stoichiometric acetylene-oxygen mixtures, highly diluted by $81-90 \%$ argon, are studied using the reactive Euler equations with a detailed chemistry model. Numerical results show that the incident Mach number $M_{0}$ changes the ODW initiation structure, giving both the smooth transition in the case of $M_{0}=10$ and the abrupt transition in the case of $M_{0}=7$. By comparing results of numerical simulation and theoretical analysis, the initiation processes are found to be chemical kineticscontrolled regardless of $M_{0}$, different from those in hydrogen-air mixtures which are wave-controlled in the low $M_{0}$ regime. The argon dilution effect on the initiation morphology is investigated, showing that the structures are determined by the dilution ratio and $M_{0}$ collectively. However, the initiation length is found to be independent of the dilution ratio and only determined by $M_{0}$, which is attributed to the competing effect of the high density and high temperature.
\end{abstract}

(c) 2019 The Combustion Institute. Published by Elsevier Inc. All rights reserved.

\section{Introduction}

In recent years, detonative combustion [1,2] has drawn increasing attention as an efficient means for hypersonic propulsion [3-6]. Among different detonation-based engine concepts, the oblique detonation has been well considered in propulsion applications such as ram accelerator and oblique detonation wave (ODW) engine.

There has been a significant progress in understanding the fundamental aspects of oblique detonation waves. In early researches, e.g., [7], analytical solutions were sought by approximating the ODW structure as an oblique shock wave (OSW) coupled with an instantaneous post-shock heat release. Later studies [8-12] demonstrate that the ODW surface is composed of a non-reactive oblique shock before the oblique detonation surface is formed, and there exists two types of OSW-ODW transition structures, as shown in Fig. 1. The former is referred to as the abrupt transition featured by a multi-wave point, while the latter is referred as the smooth transition featured by the curved shock. A number of recent studies also focused on the ODW surface instability [13-19], elucidating the formation and evolution of fine-scale unstable structures of ODWs.

\footnotetext{
* Corresponding author.

E-mail address: hhteng@bit.edu.cn (H. Teng).
}

The oblique detonation wave stems from the OSW-induced combustion process. Hence, the fuel property and its ignition influence significantly the initial ODW formation. Previous studies [20-24] on detonations in straight tubes and annular combustors (rotating detonations) demonstrated that different fuels correspond to different wave characteristics. In ODW research, one-step irreversible heat release model is widely used, in which the effects of fuel properties are overseen. Two-step induction-reaction global chemistry model has also been used to study the ODW initiation and surface instability $[25,26]$, demonstrating some intriguing phenomena that have never been observed before. Recent numerical studies based on detailed chemistry models are also performed, to look at issues such as effects of $M_{0}$ (incident Mach number) [27], inflow inhomogeneity [28,29] and initiation mechanism [30]. Our very recent study also investigates the ODW structure variation related to the effects of argon dilution in the cases under very low initial pressure $5 \mathrm{kPa}$, demonstrating a novel type of ODW structure featured by two-oblique shocks in the product [31].

In this study, the ODW structures in acetylene-oxygen mixtures highly diluted with argon are simulated. The resulting detonation in this type of combustibles corresponds usually to a very stable reaction structure. This is one kind of fuels widely used in previous detonation studies [32-34]. Equivalently, Maeda et al. [35,36] performed experimental studies on conical detonation waves by launching spherical projectiles into diluted acetylene-oxygen mixtures, illustrating different wave structures and discussing the initiation features. In this study, the simple 


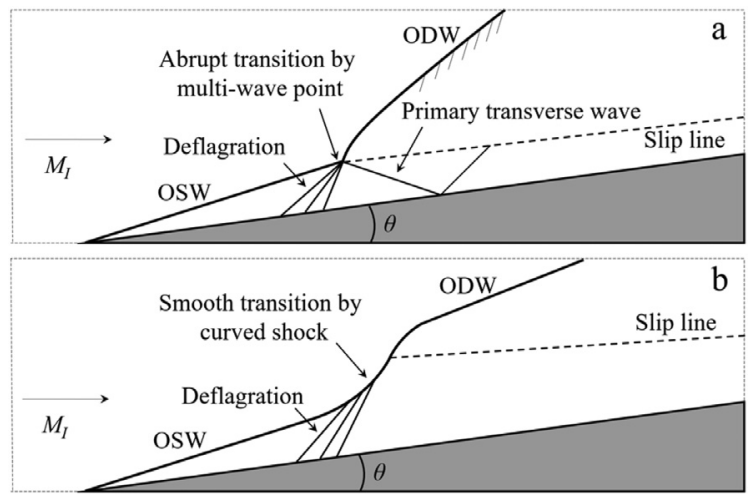

Fig. 1. Sketch of initiation structures, abrupt transition (a) and smooth transition (b).

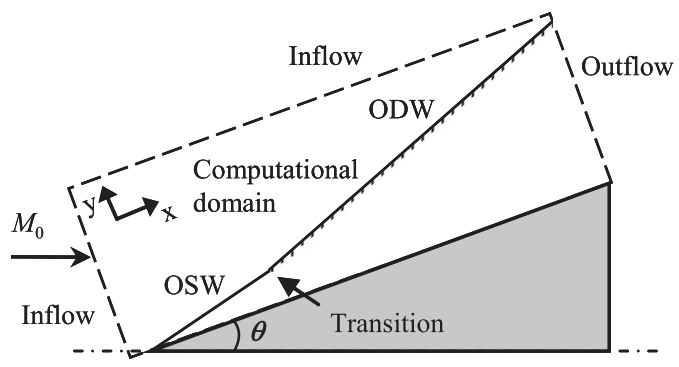

Fig. 2. Schematic of a typical ODW.

configuration of wedge-induced ODW initiation in highly argon diluted acetylene-oxygen mixtures is considered, following our recent study in hydrogen-air mixtures [30]. It is found that in the case of low $M_{0}$, the initiation mechanism is different from that in hydrogen-air mixtures, and in this paper, the resulting initiation morphology and length are discussed and analyzed in detail.

\section{Physical and mathematical models}

A schematic of a typical ODW induced by a two-dimensional (2D), semi-infinite wedge is given in Fig. 2. The presence of the wedge in a supersonic combustible inflow induces first an oblique shock wave (OSW). For a high inflow Mach number causing a high post-shock temperature behind the OSW, an exothermic chemical reaction begins, leading to the ODW formation. The coordinate is rotated to the direction along the wedge surface and the Cartesian grid in the rectangular domain enclosed by the dashed line in Fig. 2 is aligned with the wedge surface.

Following our previous studies, e.g., [27,30], the unsteady reactive Euler equations are used as governing equations for modeling the ODW flow field, i.e.:

$\frac{\partial U}{\partial t}+\frac{\partial F}{\partial x}+\frac{\partial G}{\partial y}=S$

$U=\left\{\begin{array}{c}\rho_{1} \\ \vdots \\ \rho_{n} \\ \rho u \\ \rho v \\ e\end{array}\right\}, F=\left\{\begin{array}{c}\rho_{1} u \\ \vdots \\ \rho_{n} u \\ \rho u^{2}+p \\ \rho u v \\ (e+p) u\end{array}\right\}, G=\left\{\begin{array}{c}\rho_{1} v \\ \vdots \\ \rho_{n} v \\ \rho u v \\ \rho v^{2}+p \\ (e+p) v\end{array}\right\}, S=\left\{\begin{array}{c}\omega_{1} \\ \vdots \\ \omega_{n} \\ 0 \\ 0 \\ 0\end{array}\right\}$

In the above equations, $p$ is pressure and $\rho_{i}(i=1, \ldots, n)$ is the $i$ th species density, with $u$ and $v$ denoting the velocity in the $x$ and $y$ - direction. The total density and total energy are calculated
Table 1

Chapman-Jouguet (CJ) detonation properties in different mixtures.

\begin{tabular}{lll}
\hline Gas species & $2 \mathrm{C}_{2} \mathrm{H}_{2}+5 \mathrm{O}_{2}$ with $85 \% \mathrm{Ar}$ & $2 \mathrm{H}_{2}+\mathrm{O}_{2}+3.76 \mathrm{~N}_{2}$ \\
\hline$P_{0}$ & $0.2 \mathrm{~atm}$ & $1.0 \mathrm{~atm}$ \\
$M_{C J}$ & 5.0 & 4.9 \\
$E_{a}$ & 4.8 & 7.8 \\
$\gamma_{0}$ & 1.58 & 1.40 \\
$\gamma_{C J}$ & 1.22 & 1.17 \\
$Q$ & 28.5 & 34.8 \\
\hline
\end{tabular}

by:

$\rho=\sum_{i=1}^{n} \rho_{i}, e=\rho h-p+\frac{1}{2} \rho\left(u^{2}+v^{2}\right)$

where specific enthalpy can be written as $h=\sum_{i=1}^{n} \rho_{i} h_{i} / \rho$ with $h_{i}$ obtained from the thermodynamic data of each individual species. The equation of state is

$p=\sum_{i=1}^{n} \rho_{i} \frac{R_{0}}{w_{i}} T$

where $w_{i}$ is the molecular weight, $T$ is the gas temperature, and $\omega_{i}$ is the species' specific mass production rate, which is dictated by the chemical reaction model.

The chemical kinetic model used in this study is taken from an acetylene-oxygen-diluent chemical mechanism for high-pressure ignition and detonation [37]. This detailed chemistry model involves 21 elementary reactions, 4 of which are reversible, among the 15 species. Thermodynamic properties of the chemical species are evaluated from the 9-coefficient NASA polynomial representation [38]. The governing equations are discretized on Cartesian uniform grids and solved with the DCD scheme [39] with Strang's splitting. To overcome the stiff problem, a sufficient number of sub-reaction steps are involved to assure the overall accuracy [40].

Stoichiometric acetylene-oxygen-argon mixtures, i.e., $\mathrm{C}_{2} \mathrm{H}_{2}: \mathrm{O}_{2}: \mathrm{Ar}=2: 5: \beta$, are used in which $\beta$ is determined by the dilution ratio, varying between $81 \%$ and $90 \%$ in this study. With high dilution ratios, the mixtures are stable in which the detonation structure is regular. This provides an advantage for detonation simulations avoiding numerical issues such as grid resolution and numerical stiffness. The default inflow parameters are $0.2 \mathrm{~atm}$ and $298.15 \mathrm{~K}$, and in some cases, the inflow with pressure $1.0 \mathrm{~atm}$ is also considered. Some main Chapman-Jouguet (CJ) detonation parameters in the default mixtures with $85 \%$ dilution are listed in Table 1, compared with those in stoichiometric hydrogen-air mixtures, i.e., $2 \mathrm{H}_{2}+\mathrm{O}_{2}+3.76 \mathrm{~N}_{2}$. It can be observed that CJ Mach numbers are close to each other, but other parameters are different. In general, the diluted acetylene-based mixtures are more stable with low activation energy, high specific ratio of heat capacities, and low heat release.

To set the initial conditions of simulation, the whole flow field has uniform density, velocity, and pressure, which have been calculated according to $M_{0}$ and wedge angle $\theta$, fixed at $25^{\circ}$ in all cases. Although time-dependent solvers are used in this study, all the flow fields are converged to their steady states, and unsteady processes are not considered here. The slip reflecting boundary condition is used on the wedge surface and the other boundaries are interpolated under the assumption of zero first-order derivatives of all flow parameters. On the lower computational boundary, the wedge starts from $x=0.256 \mathrm{~mm}$. Without specific description, all the length dimensions are $\mathrm{mm}$ in the following figures. Due to the multi-scale nature of the phenomena, both the computational domain and mesh scale are adjusted, and resolution studies are examined to ensure the grid-independence. 

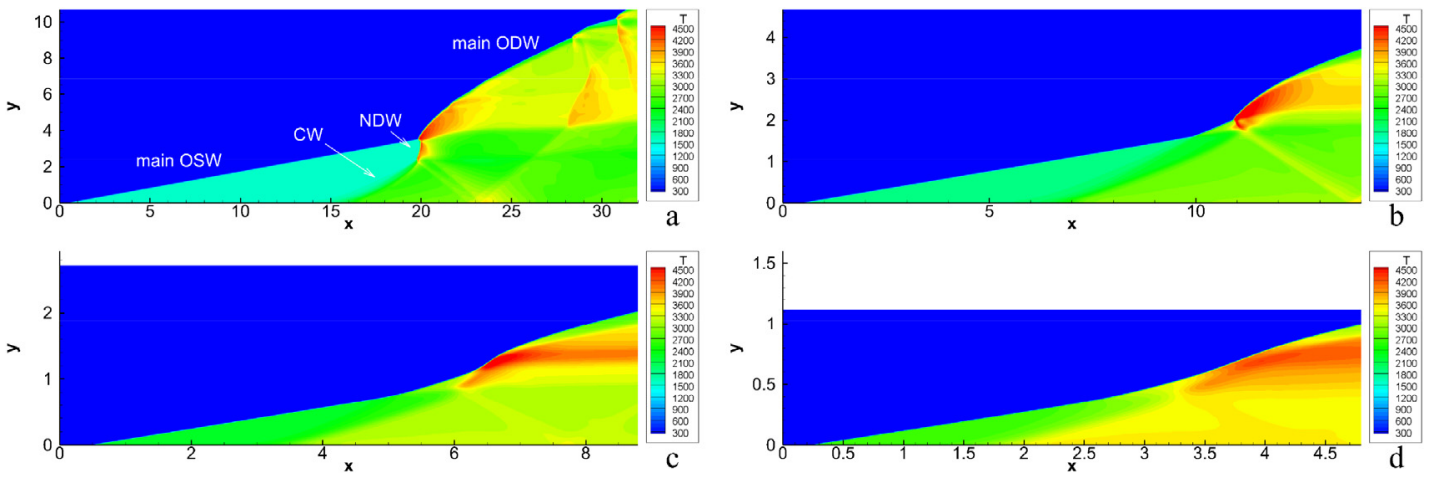

Fig. 3. Temperature fields of ODW with $85 \%$ Ar dilution, $M_{0}=7$ (a), 8 (b), 9 (c), and 10 (d) (CW: compression wave; NDW: normal detonation wave).

Table 2

Initiation lengths with different grids along several lines parallel with $x$-axis.

\begin{tabular}{lll}
\hline Position & Grid $32 \mu \mathrm{m}$ & Grid $16 \mu \mathrm{m}$ \\
\hline$x=0 \mathrm{~mm}$ & $14.71 \mathrm{~mm}$ & $14.79 \mathrm{~mm}$ \\
$x=2 \mathrm{~mm}$ & $18.98 \mathrm{~mm}$ & $19.30 \mathrm{~mm}$ \\
$x=4 \mathrm{~mm}$ & $19.88 \mathrm{~mm}$ & $20.40 \mathrm{~mm}$ \\
\hline
\end{tabular}

\section{Numerical results and discussion}

\subsection{ODW structure and resolution study}

Flow fields of ODW displayed by temperature are shown in Fig. 3. Due to the difference of inflow condition, i.e., $M_{0}$, the transition position varies in a wide range. Hence different computational domain has to be adapted to cover each corresponding complete ODW structure. The absolute grid scales for each computational domain in Fig. 3 are 32, 16, 8 and $4 \mu \mathrm{m}$, respectively. For all simulations cases, the induction region before ODW formation is resolved relatively by similar number of grid points and about 15 grids cover the half reaction zone length of each corresponding ZND detonation. It is observed that the wave structures are influenced by $M_{0}$ significantly, and the initiation position moves upstream when $M_{0}$ increases. The abrupt transition of OSW-ODW is observed in Fig. 3a, resulting in a complicated wave structure. Beneath the multi-wave point, a short section of normal detonation wave (NDW) is generated, which connects a compression wave (CW) extending to the wedge. Increasing $M_{0}$ to 8 , the NDW disappears while a curved oblique shock appears before the multi-wave point, indicating the onset from the abrupt to smooth transition. Increasing $M_{0}$ further to 9 and 10, the multi-wave point disappears and the transition is smooth by a curved shock, as shown in Fig. 3c and d.

To assess the effects of grid scale, a resolution study is performed by doubling the grid number in each direction. Results of $M_{0}=7$ and 10 are shown by contours in Fig. 4, compared with those obtained using the default resolution. In the case of $M_{0}=7$, the wave structure is similar to each other, although minor effects of high resolution can be noticed. With a higher resolution, the multi-wave point moves just slightly downstream, and the reflected shock wave in the combustion product is more apparent. The results of $M_{0}=10$ are in better agreement and almost all the contours overlap with each other shown in Fig. 4b, demonstrating the results approach grid-independent. To quantify effects of different grids, we use the temperature increase of $10 \%$ to define the initiation position along different lines parallel to the $x$-axis. Results based on different grids are shown in Table 2, demonstrating that the grid has little effect on the position. In fact, the maximum
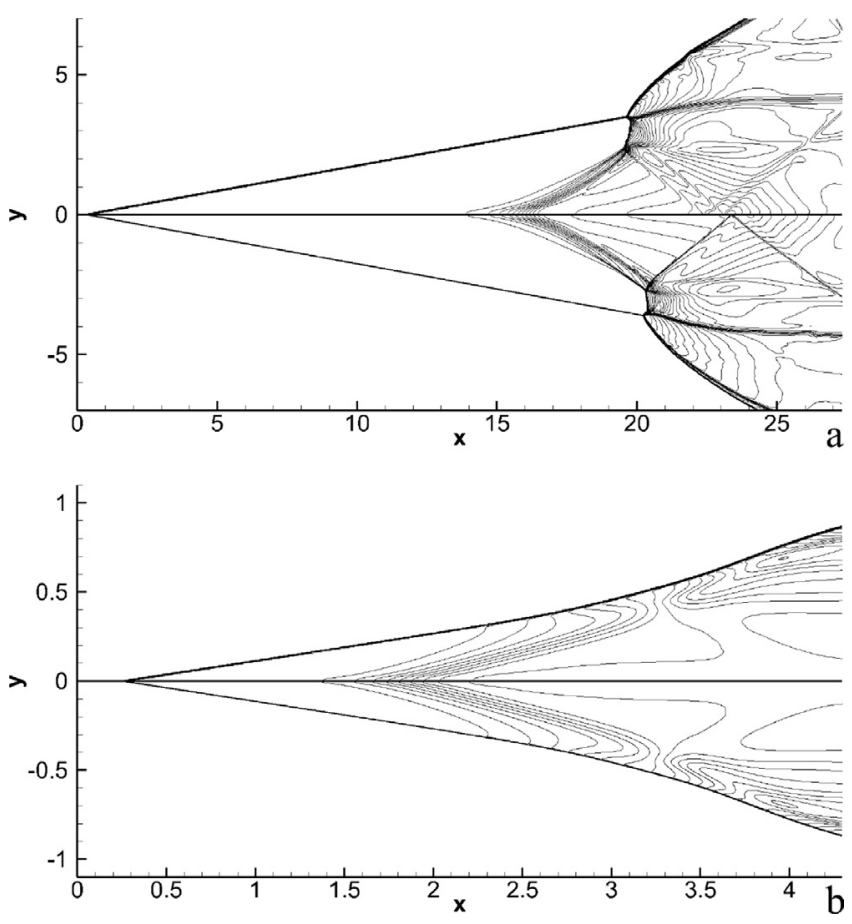

Fig. 4. Temperature shown by contours with $85 \% \operatorname{Ar}$ dilution, (a) $M_{0}=7$, grid $32 \mu \mathrm{m}$ (upper) and $16 \mu \mathrm{m}$ (lower); (b) $M_{0}=10$, grid $4 \mu \mathrm{m}$ (upper) and $2 \mu \mathrm{m}$ (lower).

difference appears around the multi-wave point, about $0.7 \mathrm{~mm}$. Considering the length from the wedge tip to the multi-wave point is about $20 \mathrm{~mm}$, the discrepancy from the grid effect is about $3.5 \%$. This small discrepancy appears to be acceptable, and the grid scale of $32 \mu \mathrm{m}$ is thus deemed sufficient and used for all cases in the following analysis.

\subsection{Comparison between numerical and theoretical analysis}

Previous study [30] has looked at the ODW structures in hydrogen-air mixtures, which are similar to those shown in Figs. 3 and 4. However, the results of [30] conjecture that there are two kinds of initiation mechanism in ODW, one is chemical kinetics-controlled and the other is wave- (or gasydnamics-) controlled. Determining the mechanism is achieved by analyzing the fluid elements in the vicinity of the wedge surface. In the kineticscontrolled one, the initiation length, roughly the position of initiation structure, can be predicted by constant volume combustion (CVC) theory. Contrarily, in the wave-controlled ODWs, the initiation length is usually much shorter than that predicted by the 


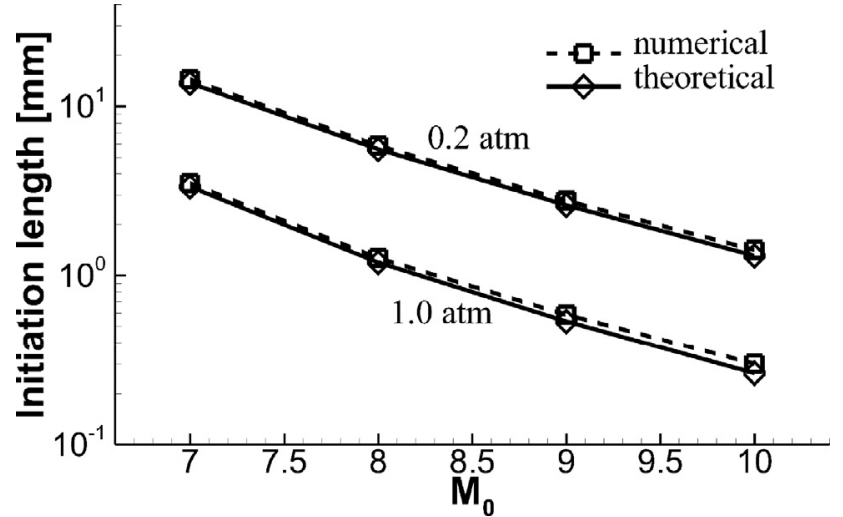

Fig. 5. Numerical vs. theoretical initiation lengths in the $85 \%$ Ar diluted mixtures.

CVC theory, and there is still lack of theoretical approach to predict this length. It is shown that in hydrogen-air mixtures, the wavecontrolled gasdynamic process occurs in the case of low $M_{0}$ with the abrupt transition (as sketched in Fig. 1a), while the chemical kinetics-controlled mechanism occurs in the case of high $M_{0}$ with the smooth transition (as sketched in Fig. 1b). However, it is still unclear whether these mechanisms are universal and applicable in other combustible mixtures.

This study aims to explore the initiation mechanisms in stoichiometric acetylene-oxygen mixtures with high degree of argon dilution. The numerical and theoretical initiation lengths introduced in [30] are employed. The numerical initiation length is defined along the flow stream direction, parallel with the $x$-axis, from the wedge tip to the end of induction zone, i.e., the location at which the temperature increases over $10 \%$ in this study. Correspondingly, a theoretical approach based on the CVC theory is used to define the theoretical initiation length. It assumes an ideal postoblique shock flow, where the mixture is completely burned, and pressure build-up and the formation of pressure waves are weak near the wedge. Under these assumptions, calculations can be performed using the CHEMKIN package [41]. The inputs of CVC calculations include species densities and temperature, which can be determined by either oblique-shock relations or numerical simulations. It is found that the former does not give the right results because the ratio of specific heat changes after the strong oblique shock and hence, the numerical results are used in this study. Postoblique-shock species densities and temperature are then used to simulate CVC to obtain the reaction time required to attain a mixture temperature with a $10 \%$ increase from its post-shock value. The theoretical initiation length is deduced by multiplying the time with the post-oblique-shock particle velocity. Despite its simple formulation, this analysis provides a predictive approach for the general structure of oblique detonations. It is worth mentioning that other criteria can be used to define the end of induction zone (hence the initiation length). Nevertheless, if both theoretical and numerical results take on the same criterion, the results would not be affected. Furthermore, the temperature increment of $10 \%$ can be altered in a range, and tests have found that the choice of $5 \%$ is still valid giving the same qualitative results. However, the value cannot be changed too large, e.g., $20 \%$, otherwise a large part of heat release zone is included, violating the intent to define the induction zone.

The initiation length results are plotted in Fig. 5, including both the numerical and theoretical ones. It is observed that the numerical initiation lengths depend on the pressure and $M_{0}$, and the curve for the same pressure is almost a straight line with the logarithmic $y$ - axis coordinate. However, the differences between numerical and theoretical results are very small, and the deviation of
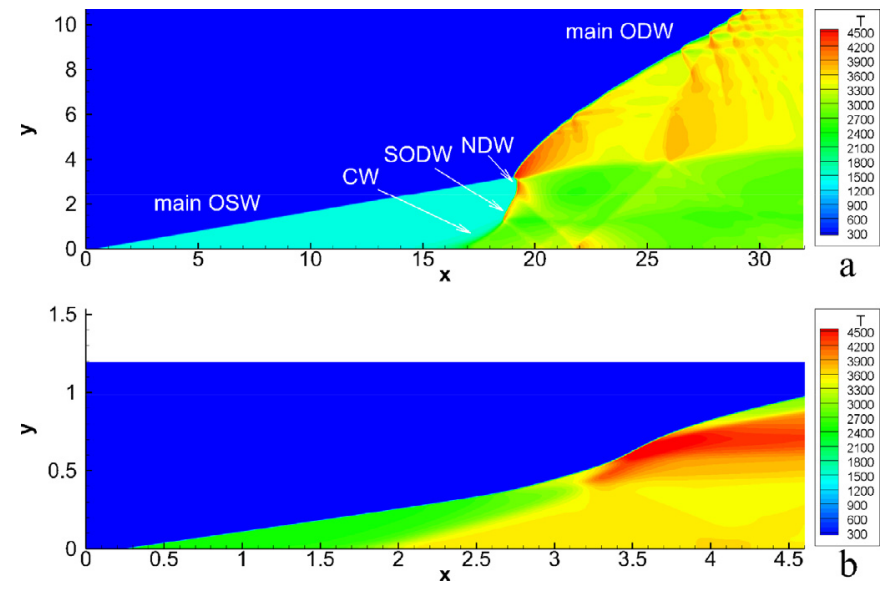

Fig. 6. Temperature fields of ODW with $\mathrm{Ar}$ ratio $81 \%$ and $M_{0}=7$ (a) and 10 (b) (CW: compression wave; SODW: secondary ODW; NDW: normal detonation wave).
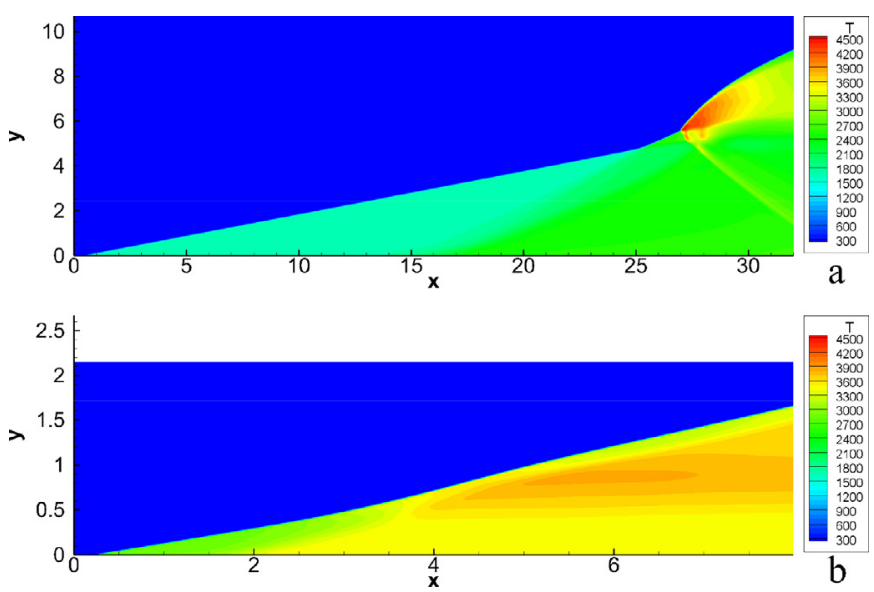

Fig. 7. Temperature fields of ODW with $90 \%$ Ar dilution and $M_{0}=7$ (a) and 10 (b).

numerical and theoretical lengths are not prominently observed, different from those in hydrogen-air mixtures [30]. As pointed out before, in the cases of low $M_{0}$, i.e., 7 , the self-ignition induced by OSW and subsequent detonation afterward does not occur, so the initiation lengths are determined by the shock and detonation wave interaction within the initiation region, called wavecontrolled mechanism. Nevertheless, from the results in Fig. 5, the self-ignition governed by chemical kinetics dominates all the cases so the initiation remains chemical kinetics-controlled regardless of $M_{0}$ in acetylene-oxygen mixtures highly diluted with argon.

\subsection{Effects of argon dilution}

To clarify the initiation mechanism of stoichiometric acetyleneoxygen mixtures with high argon dilution, simulations with different Ar ratios are performed and results are shown in Figs. 6 and 7. In general, the structures with $M_{0}=10$ are similar, as shown in Figs. $6 \mathrm{~b}$ and $7 \mathrm{~b}$. However, the structures with $M_{0}=7$ are different and influenced by the Ar dilution ratio. Aforementioned structure in Fig. 3a shows a formation with the connected NDW and CW. Decreasing Ar dilution ratio to $81 \%$, the structure becomes more complex. A secondary ODW (SODW) appears between the NDW and CW. Two parallel oblique shock waves are observed in the combustion product, one is from the connection of CW and SODW, and the other is from the connection of SODW and NDW, as shown in Fig. 6a. Increasing Ar dilution to 90\%, the structure becomes simple, and only a train of CW is observed in Fig. 7a. 

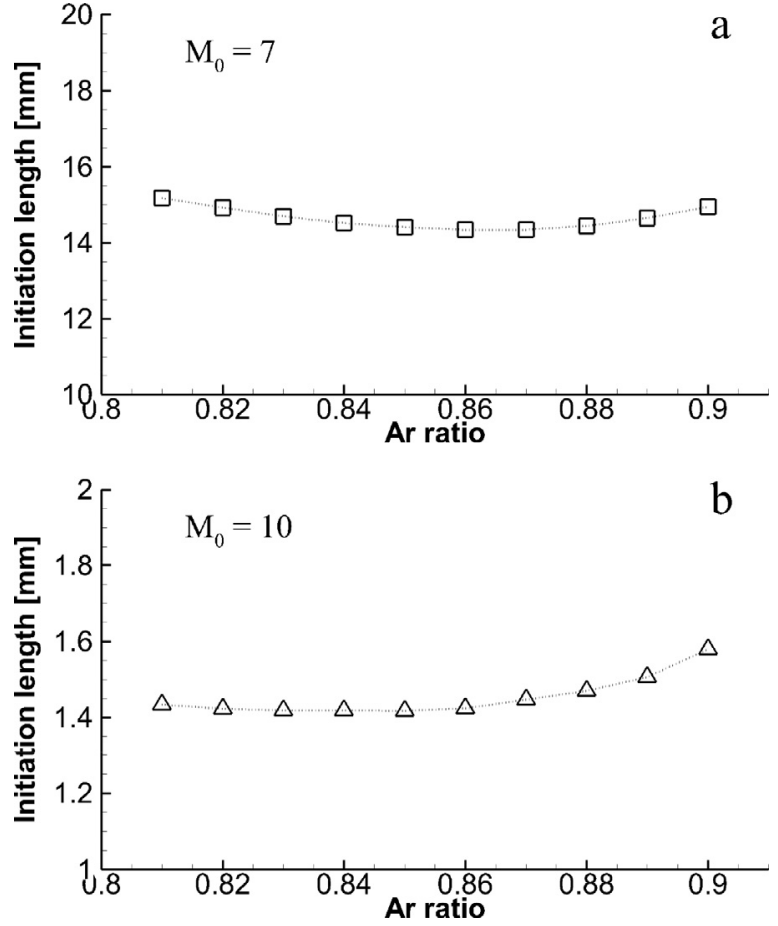

Fig. 8. Initiation lengths as function of Ar dilution ratio in the cases of $M_{0}=7$ and 10 .

Numerical initiation lengths are plotted in Fig. 8 for further quantitative analysis. The figure shows that the initiation lengths are insensitive to Ar ratio in both high and low $M_{0}$ cases. It is important to point out that the theoretical results are very close to the numerical ones. Hence, the former are not displayed here. In Fig. 8, it is observed that the lengths are about $14 \sim 15$ with $M_{0}=7$, while about 1.5 with $M_{0}=10$. For both $M_{0}$ cases, a slight initiation length variation can be observed, i.e., the length first decreases and then increases when $\mathrm{Ar}$ ratio increases, but the variation is relatively small so that the initiation length can be considered approximately as independent of $\mathrm{Ar}$ ratio.

\subsection{Discussion on the initiation characteristics}

It is interesting to observe that initiation lengths are insensitive to $\mathrm{Ar}$ ratio. From the viewpoint of fuel, i.e., acetylene-oxygen, the variation of Ar ratio from $81 \%$ to $90 \%$ is not a minor variation. Indeed, the fuel ratio decreases from $19 \%$ to $10 \%$ in this process, a very significant variation, so the near-constant initiation length needs to be clarified. Therefore, pressure and temperature along the lines $y=0 \mathrm{~mm}$ in the case of $M_{0}=7$ are plotted in Fig. 9. It is observed that before $x=15 \mathrm{~mm}$, the post-oblique shock pressure values are close in both cases, but the case with $90 \%$ Ar has a higher post-oblique shock temperature than that with $81 \% \mathrm{Ar}$. Relatively high temperature in the case of $90 \%$ Ar should be derived from the species properties. As one kind of monatomic molecule, Ar has a high ratio of specific heat capacities. This results in the high temperature of the mixtures with $90 \% \mathrm{Ar}$, as shown by the red solid curve in Fig. 9. High temperature facilitates the ignition and accelerates the induction process, subsequently the heat release is easier to be triggered. However, the case with $81 \% \mathrm{Ar}$ ratio has higher fuel density, accelerating the induction process from another way, and finally a rapid increase of temperature can be observed shown by the red dashed curve in Fig. 9. Overall, high temperature and high density play their roles in the high and low $\mathrm{Ar}$ dilution cases, respectively. In other words, these two factors from
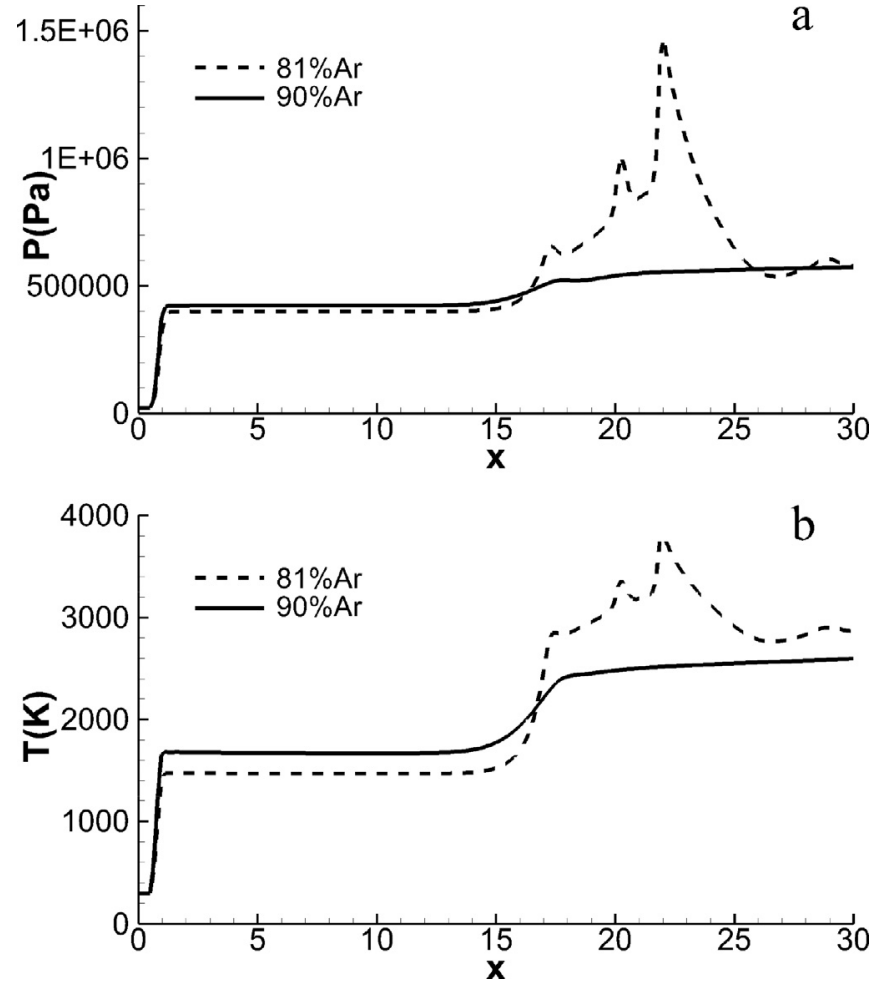

Fig. 9. Pressure and temperature along the lines $y=0$ with $M_{0}=7$.

including more Ar compensate each other, so the initiation lengths are insensitive to the dilution ratio.

Compared with the previous study based on stoichiometric hydrogen-air mixtures [30], more understanding on the initiation of wedge-induced ODW can be achieved. The results shown in Fig. 9 provides an explanation of why the initiation is chemical kinetics-controlled in both high and low $M_{0}$ cases. It is observed that the post-oblique shock temperature is about $1470 \mathrm{~K}$ in the $81 \%$ Ar ratio case, and about $1670 \mathrm{~K}$ in the $90 \%$ Ar case. These temperature values are much higher than that in hydrogen-air mixtures, about $1100 \mathrm{~K}$ in the case of $M_{0}=7$. Both studies indicate that the temperature is strongly dependent on $M_{0}$. Decreasing $M_{0}$ makes the initiation mechanism changing from kinetics-controlled to wave- (gasydynamic-) controlled, and also results in a change of ODW morphology, from smooth to abrupt. Based on the results of previous study, i.e., ODWs in stoichiometric hydrogen-air mixtures, it is skeptical that the ODW morphology determines the initiation mechanism. However, in the present study using acetylene-oxygen-argon mixtures, various ODW configurations appear under the kinetics-controlled cases. Therefore, for the wedgeinduced ODW formation, the initiation mechanism cannot be determined by ODW morphology, and the thermodynamic state behind the post-oblique shock, e.g., temperature or density, appears to be a more important factor.

\section{Conclusions}

Oblique detonation waves in stoichiometric acetylene-oxygen mixtures with $81 \%$ to $90 \%$ argon dilution are simulated with a detailed chemistry model. As a common fuel used in many detonation research, there remain research questions concerning the initiation mechanism of acetylene-fueled ODWs. The present simulation results are analyzed and further compared with ODWs in stoichiometric hydrogen-air mixtures, revealing some special features and benefiting to the knowledge of ODW initiation independent of specific mixtures. 
Numerical results show that the incident Mach number $M_{0}$ changes the initiation structure, but by comparing the numerical simulation and theoretical analysis results, the initiation mechanism is found to be chemical kinetics-controlled in both high and low $M_{0}$ cases, different from that in hydrogen-air mixtures. The effect of argon dilution on the initiation morphology is investigated, showing that the structure is sensitive only in the cases of low $M_{0}$, and different structures are observed by changing the argon dilution ratio. However, the initiation length remains independent against $\mathrm{Ar}$ dilution ratio and is only determined by $M_{0}$, which is attributed to the competition of the high density and high temperature. By comparing with the previous study based on stoichiometric hydrogen-air mixtures, the initiation mechanism is found to be determined mainly by the temperature, and not the ODW morphology in highly argon diluted acetylene-oxygen mixture.

As a first step in extending the investigation of wedge-induced acetylene ODW, this study employs acetylene-oxygen mixtures with high amount of argon dilution. Although CJ Mach numbers of these mixtures are close to those of stoichiometric hydrogenair mixtures, other detonation-related parameters, such as energy release, differ from each other. These parameters are inter-related by the gas thermodynamic properties, hence it is impossible to change only one parameter while keeping the others fixed. Effects of compressibility, energy release, and reaction sensitivity collectively change the structure and initiation [42-44], and more work on each factor should be performed in the future.

\section{Acknowledgments}

The research is supported by the National Natural Science Foundation of China, NSFC (nos. 11822202 and 91641130) and the Natural Sciences and Engineering Research Council of Canada, NSERC (no. RGPIN-2017-06698).

\section{References}

[1] W. Fickett, W.C. Davis, Detonation: Theory and Experiment (Dover Books on Physics), Dover Publications, New York, 2000.

[2] J.H.S. Lee, The Detonation Phenomenon, Cambridge University Press, New York, 2008

[3] G.D. Roy, S.M. Frolov, A.A. Borisov, D.W. Netzer, Pulse detonation propulsion: challenges, current status, and future perspective, Prog. Energy Combust. Sci. 30 (2004) 545-672.

[4] P. Wolanski, Detonation propulsion, Proc. Combust. Inst. 34 (2013) 125-158.

[5] E.M. Braun, F.K. Lu, D.R. Wilson, J.A. Camberos, Airbreathing rotating detonation wave engine cycle analysis, Aerosp. Sci. Technol. 27 (2013) 201-208.

[6] F.K. Lu, H. Fan, D.R. Wilson, Detonation waves induced by a confined wedge, Aerosp. Sci. Technol. 10 (8) (2006) 679-685.

[7] D.T. Pratt, J.W. Humphrey, D.E. Glenn, Morphology of standing oblique detonation waves, J. Propul. Power 7 (5) (1991) 837-845

[8] C. Li, K. Kailasanath, E.S. Oran, Detonation structures behind oblique shocks, Phys. Fluids 6 (1994) 1600-1611.

[9] C. Viguier, L.F. Figueira da Silva, D. Desbordes, B. Deshaies, Onset of oblique detonation waves: comparison between experimental and numerical results for hydrogen-air mixtures, Symp. (Int.) Combust. 26 (2) (1996) 3023-3031.

[10] L.F. Figueira da Silva, B. Deshaies, Stabilization of an oblique detonation wave by a wedge: a parametric numerical study, Combust. Flame 121 (2000) $152-166$

[11] H.H. Teng, Z.L. Jiang, On the transition pattern of the oblique detonation structure, J. Fluid Mech. 713 (2012) 659-669.

[12] T. Wang, Y. Zhang, H. Teng, Z. Jiang, H.D. Ng, Numerical study of oblique detonation wave initiation in a stoichiometric hydrogen-air mixture, Phys. Fluids 27 (2015) 096101.

[13] J.Y. Choi, D.W. Kim, I.S. Jeung, F. Ma, V. Yang, Cell-like structure of unstable oblique detonation wave from high-resolution numerical simulation, Proc. Combust. Inst. 31 (2007) 2473-2480.

[14] J. Verreault, A.J. Higgins, R.A. Stowe, Formation of transverse waves in oblique detonations, Proc. Combust. Inst. 34 (2) (2013) 1913-1920.

[15] H.H. Teng, Z.L. Jiang, H.D. Ng, Numerical study on unstable surfaces of oblique detonations, J. Fluid Mech. 744 (2014) 111-128.
[16] H. Teng, H.D. Ng, K. Li, C. Luo, Z. Jiang, Evolution of cellular structures on oblique detonation surfaces, Combust. Flame 162 (2015) 470-477.

[17] P. Yang, H.D. Ng, H. Teng, Z. Jiang, Initiation structure of oblique detonation waves behind conical shocks, Phys. Fluids 29 (8) (2017) 086104

[18] Z. Ren, B. Wang, G. Xiang, L. Zheng, Effect of the multiphase composition in a premixed fuel-air stream on wedge-induced oblique detonation stabilisation, J. Fluid Mech. 846 (2018) 411-427.

[19] S. Miao, J. Zhou, Z. Lin, X. Cai, S. Liu, Numerical study on thermodynamic efficiency and stability of oblique detonation waves, AIAA J. 56 (2018) 3112-3122.

[20] B. Zhang, The influence of wall roughness on detonation limits in hydrogen-oxygen mixture, Combust. Flame 169 (2016) 333-339.

[21] B. Zhang, H. Liu, B.J. Yan, Effect of acoustically absorbing wall tubes on the near-limit detonation propagation behaviors in a methane-oxygen mixture, Fuel 236 (2019) 975-983.

[22] B. Zhang, H. Liu, B.J. Yan, Investigation on the detonation propagation limit criterion for methane-oxygen mixtures in tubes with different scales, Fuel 239 (2019) 617-622

[23] Z. Pan, B. Fan, X. Zhang, M. Gui, G. Dong, Wavelet pattern and self-sustained mechanism of gaseous detonation rotating in a coaxial cylinder, Combust. Flame 158 (2011) 2220-2228.

[24] Z. Pan, J. Qi, J. Pan, P. Zhang, Y. Zhu, M. Gui, Fabrication of a helical detonation channel: effect of initial pressure on the detonation propagation modes of ethylene/oxygen mixtures, Combust. Flame 192 (2018) 1-9.

[25] P. Yang, H. Teng, H.D. Ng. Z. Jiang, A numerical study on the instability of oblique detonation waves with a two-step induction-reaction kinetic model, Proc. Combust. Inst. 37 (2019) 3537-3544.

[26] P. Yang, H. Teng, Z. Jiang, H.D. Ng, Effects of inflow Mach number on oblique detonation initiation with a two-step induction-reaction kinetic model, Combust. Flame 193 (2018) 246-256.

[27] H. Teng, Y. Zhang, Z. Jiang, Numerical investigation on the induction zone structure of the oblique detonation waves, Comput. Fluids 95 (2014) 127-131.

[28] Y. Fang, Z. Hu, H. Teng, Z. Jiang, H.D. Ng, Effects of inflow equivalence ratio inhomogeneity on oblique detonation initiation in hydrogen-air mixtures, Aerosp. Sci. Technol. 71 (2017) 256-263.

[29] K. Iwata, S. Nakaya, M. Tsue, Wedge-stabilized oblique detonation in an inhomogeneous hydrogen-air mixture, Proc. Combust. Inst. 36 (2) (2017) 2761-2769.

[30] H. Teng, H.D. Ng, Z. Jiang, Initiation characteristics of wedge-induced oblique detonation wave in a stoichiometric hydrogen-air mixture, Proc. Combust. Inst 36 (2) (2017) 2735-2742.

[31] Y. Fang, Y. Zhang, X. Deng, H. Teng, Numerical study of wedge-induced oblique detonation in acetylene-oxygen- argon mixtures, Phys. Fluids 31 (2019) 026198

[32] J.H.S. Lee, Dynamic parameters of gaseous detonations, Ann. Rev. Fluid Mech. 16 (1984) 311-336.

[33] M.I. Radulescu, H.D. Ng, J.H.S. Lee, B. Varatharajan, The effect of argon dilution on the stability of acetylene/oxygen detonations, Proc. Combust. Inst. 29 (2) (2002) 2825-2831.

[34] B. Zhang, N. Mehrjoo, H.D. Ng, J.H.S. Lee, C. Bai, On the dynamic detonation parameters in acetylene-oxygen mixtures with varying amount of argon dilution, Combust. Flame 161 (5) (2014) 1390-1397.

35] S. Maeda, J. Kasahara, A. Matsuo, Oblique detonation wave stability around spherical projectile by a high time resolution optical observation,, Combust. Flame 159 (2) (2012) 887-896

[36] S. Maeda, S. Sumiya, J. Kasahara, A. Matsuo, Initiation and sustaining mechanisms of stabilized oblique detonation waves around projectiles, Proc. Combust. Inst. 34 (2) (2013) 1973-1980.

[37] B. Varatharajan, F.A. Williams, Chemical-kinetic descriptions of high-temperature ignition and detonation of acetylene-oxygen-diluent systems, Combust. Flame 124 (4) (2001) 624-645.

[38] B.J. McBride, M.J. Zehe, S. Gordon, NASA Glenn coefficients for calculating thermodynamic properties of individual species, Report No. NASA/TP-2002-211556, NASA Glenn Research Center, Cleveland, USA, 2002.

[39] Z. Jiang, On dispersion-controlled principles for non-oscillatory shock-capturing schemes, Acta Mech. Sin. 20 (1) (2004) 1-15.

[40] H.C. Yee, D.V. Kotov, W. Wang, C.W. Shu, Spurious behavior of shock-capturing methods by the fractional step approach: problems containing stiff source terms and discontinuities, J. Comput. Phys. 241 (2013) 266-291.

[41] R.J. Kee, F.M. Rupley, E. Meeks, J.A. Miller, CHEMKIN-III: a fortran chemical kinetic package for the anaylsis of gas-phase chemical and plasma knietics, Report No. SAND-96-8216, Sandia National Labs, Livermore, CA, USA, 1996.

[42] V.I. Manzhalei, Fine structure of the leading front of a gas detonation, Combust. Explos. Shock Waves 13 (3) (1977) 402-404.

[43] P. Mach, M.I. Radulescu, Mach reflection bifurcations as a mechanism of cell multiplication in gaseous detonations, Proc. Combust. Inst. 33 (2) (2011) 2279-2285.

[44] M.I. Radulescu, A detonation paradox: why inviscid detonation simulations predict the incorrect trend for the role of instability in gaseous cellular detonations? Combust. Flame 195 (2018) 151-162. 